\title{
A new denoising technique for ultrasound images using morphological properties of speckle combined with tissue classifying parameters
}

\author{
Gjenna Stippel $^{a}$, Wilfried Philips ${ }^{a}$, Ignace Lemahieu ${ }^{b}$ \\ ${ }^{a}$ Ghent University, Dept. TELIN, Ghent, Belgium \\ ${ }^{b}$ Ghent University, Dept. ELIS, Ghent, Belgium
}

\begin{abstract}
In this paper we introduce a new speckle suppression technique for medical ultrasound images that incorporates morphological properties of speckle as well as tissue classifying parameters. Each individual speckles is located, and, exploiting our prior knowledge on the tissue classification, it is determined whether this speckle is noise or a medically relevant detail.

We apply the technique on images of neonatal brains affected by White Matter Damage (leukomalacia). The results show that applying an active contour on a processed image, in order to segment the affected areas, yields a segmentation much closer to that of an expert.
\end{abstract}

Keywords: medical ultrasound, speckle, texture, morphology, tissue classification

\section{INTRODUCTION}

Ultrasound imaging is gaining more and more importance in medical practice nowadays. It is especially useful in imaging soft tissue like liver, spleen, lungs, the heart, and the neonatal brain. Advantages of ultrasound imaging are that it is quick, cheap, and the machinery is highly portable. An ultrasound image can be made in a few seconds, while standing next to the bed of the patient. This is especially useful when the patient is in need of intensive care, as is usually the case with prematurely born infants.

A common problem with the interpretation of ultrasound images though, is the presence of speckle noise. Several techniques for suppressing speckle noise have been developed. ${ }^{1-6}$ They can be divided into two classes:

1. Techniques that are applied directly in the original image domain like the Lee ${ }^{1}$ and the Frost ${ }^{2}$ filters,

2. Techniques that are applied in the wavelet domain like the technique developed by Malfait and Roose, ${ }^{3}$ which is a universal filter, and the technique proposed by Sattar, Floreby, Salomonsson and Lövström, ${ }^{4}$ designed especially for speckle noise reduction.

None of these methods take into account any morphological properties of speckle; neither do they incorporate any prior knowledge concerning the different speckle statistics in the different tissues. This second thing makes them less suitable to enhance specific (desired) medical features in the image.

In this article we focus on ultrasound images of the neonatal brain, and in particular on the disease "White Matter Damage" (leukomalacia). White Matter Damage (WMD) is found with $20 \%$ to $50 \%$ of all neonates of

Further author information: (Send correspondence to Gjenna Stippel)

Gjenna Stippel: E-mail: Gjenna.Stippel@telin.rug.ac.be, Telephone: +32 9 2643426, Fax: +32 92644295

URL: http://telin.rug.ac.be/ gs or http://www.gjenna.com

Wilfried Philips: E-mail: philips@telin.rug.ac.be, Address: Ghent University, Department of Telecommunications and Information Processing (TELIN), St.-Pietersnieuwstraat 41, 9000, Ghent, Belgium

Ignace Lemahieu: E-mail: Ignace.Lemahieu@rug.ac.be, Address: Ghent University, Department of Electronics and Information Systems (ELIS), St.-Pietersnieuwstraat 41, 9000, Ghent, Belgium 


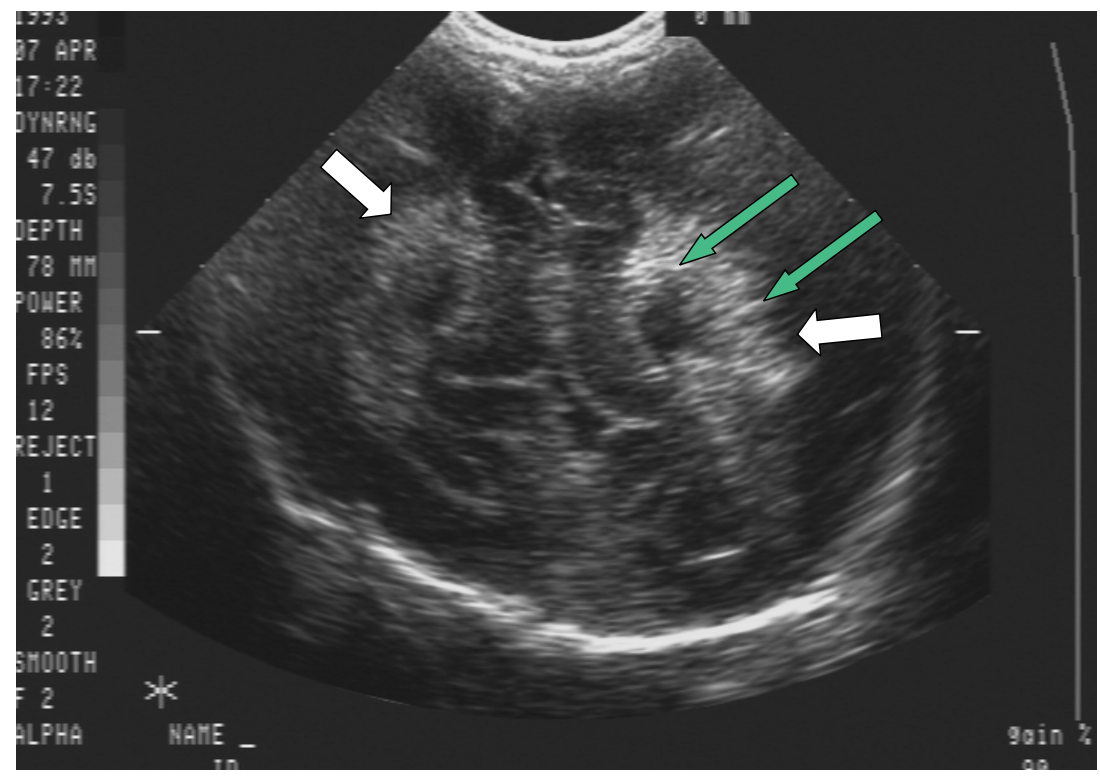

Figure 1: Flares and "accents".

very low birth weight (i.e., less than $1500 \mathrm{~g}$ ). It is visible in an ultrasound image of the brain as "white clouds" (so-called "flares", see the white arrows in figure 1).

Three features are most important in determining the gravity of the damage: the shape and the area of the flares, and the presence (or absence) of "accents", i.e., particularly bright speckles that are bigger than usual, located within the flares (e.g. the ones pointed at by the long thin arrows in figure 1). ${ }^{7,8}$

The basic idea behind the technique proposed in this paper is to segment all speckles separately, after which we filter each speckle adaptively, dependent of the texture characteristics of its immediate vicinity. The segmentation itself consists of a region growing procedure controlled by the grey-values of the pixels. In order to steer this process with respect to the shape of the regions to be grown, we pre-process the image morphologically, using an "ideal speckle" as structure element.

We conclude with segmenting the flares on the filtered images with the help of an active contour. Comparison of the results with the manually drawn segmentations of an expert will reveal a considerable improvement of the performance of the active contour over its performance on the unprocessed image as well as on the image, processed with one of the classical techniques.

\section{PREPARATORY WORK}

In this section we introduce the two basic elements that form the heart of the proposed technique: the tissue classifying parameters and the morphological structure element.

\subsection{Tissue Classifying Parameters}

As explained in the introduction, once we have segmented all speckles individually, we classify them by considering the texture characteristics of its immediate vicinity. To obtain those distinguishing parameters, we performed the following experiment:

When making an ultrasound image of a neonatal brain the neonatologist can select various scanner settings, like the power (the amplitude of the emitted waves), the gain (the overall amplification of the received signal), the Time Gain Compensation (different levels of amplification for the received signals from different depths) etc. Since we want to quantitatively compare images with respect to first and second order texture statistics, (which are obviously influenced by these manual scanner settings), we have to construct "standard images" first, which 

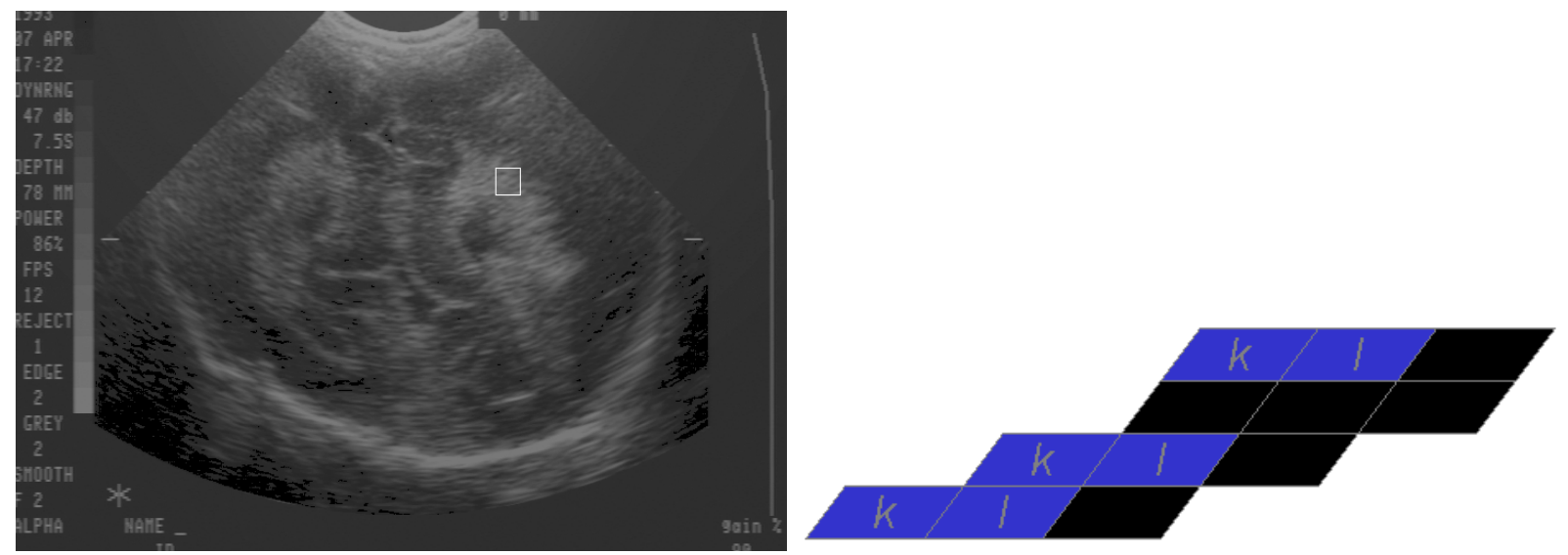

Figure 2: Left: The specific rectangle (simulation), Right: a small region.

are independent of those scanner settings. This problem has been studied extensively by Simaeys et al. ${ }^{9}$ and a compensation algorithm that constructs such standard image is described. In figure 2 the compensated version of the image in figure 1 is shown.

We considered 48 images of neonates, all of which were classified by the neonatologist as certainly ill (i.e., suffering from leukomalacia) or certainly healthy. All of these images were processed by the compensation algorithm first. In the resulting 48 compensated images we selected a rectangle of $30 \times 30$ pixels at exactly the same spot (from an anatomical viewpoint) as demonstrated in figure 2 . According to the neonatologist, there is certainly a flare present in that region, if the infant suffers from leukomalacia.

Within the rectangle we calculated several parameters including the mean grey-value and the contrast. This "contrast" is defined as follows: let $r$ be a region in the image like, for instance, the one shown in figure 2. Denote by $A_{k l}$ the number of pairs of adjacent pixels within $r$ with grey-values $k$ and $l$ respectively. (In our example $\left.A_{k l}=3\right)$.

Now we define the contrast $\gamma_{r}$ of $r$ as:

$$
\gamma_{r}=\frac{\sum_{k, l=0}^{255}(k-l)^{2} A_{k l}}{\sum_{k, l=0}^{255} A_{k l}}
$$

The contrast is a measure for how many grey-value transitions there are in the region under consideration; the more adjacent pixels with a big difference in grey-value there are in $r$, the higher the contrast $\gamma_{r}$ is. In practice, the contrast is calculated by means of the cooccurrence matrix. ${ }^{10-12}$

The mean grey-value and the contrast turn out to be distinctive in determining whether the area under consideration is ill or healthy. A scatter plot of the results is shown in figure 3 . The separate cluster in the bottom left corner indicates that a mean grey-value of less than 67, and a contrast of less than 35 means that the tissue within the area is healthy, otherwise it is ill. Similar results, but for ultrasound images of the prostate, were obtained in literature. ${ }^{10,13,14}$

\subsection{Morphological Structure Element}

The segmentation of the individual speckles is achieved by a region growing procedure based on the grey-values of the pixels. In order to regulate the shapes of the regions grown, we pre-process the image with a sequence of morphological operations, using a morphological structure element, which thereby serves as an "ideal speckle" (see section 3 for a detailed explanation of those morphological operations).

Speckle is visible in ultrasound images as short, slightly "banana-shaped" white lines. This is a result of the properties of the sound beam together with those of the imaging equipment. If we restrict ourselves to 


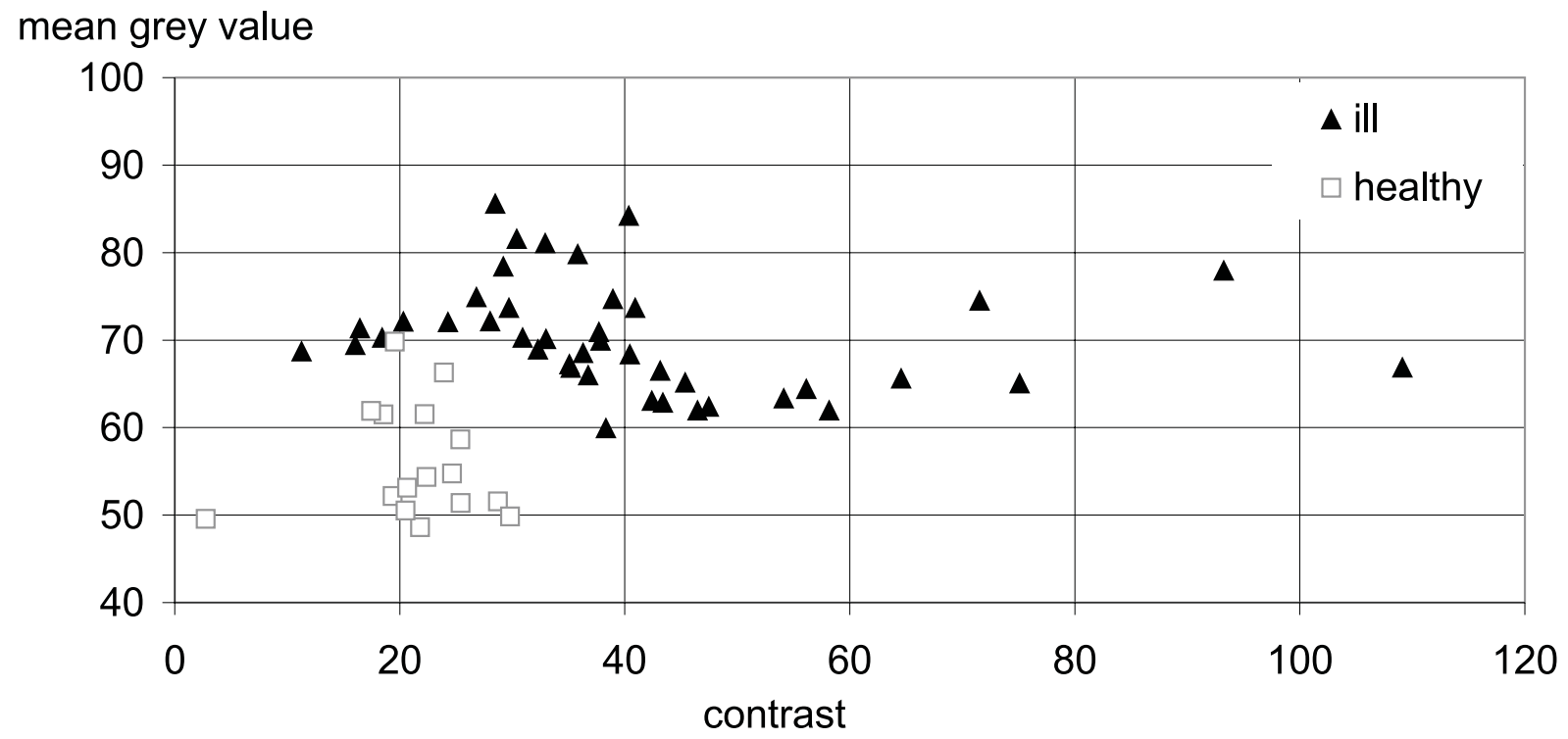

Figure 3: Measured texture characteristics.

the focal zone of the sound field and furthermore assume that the number density of the scattering structures is sufficiently high to yield so-called fully developed speckle, then there have been derived analytic formulas for the axial and lateral size of the speckle by Wagner et al. ${ }^{15}$ According to their results the axial speckle size (i.e., in the direction of the sound beam) is exclusively and inversely dependent on the bandwidth of the employed transducer, i.e., proportional to the spatial pulse length. The lateral speckle size (i.e., perpendicular to the sound beam), in creases proportional to the depth. If however we look outside the focal zone, or when the number density of the scatterers is low, then the lateral and axial size of the speckle cannot be analytically calculated. ${ }^{16}$

Since all images we investigated had been taken with the sane ultrasound scanner, and with the same central frequency of $7.5 \mathrm{MHz}$ the axial size of the speckles is the same in all images. Furthermore, the flares are located at approximately the same place in all images, so also the lateral speckle size is the approximately the same in our region of interest in all images.

Exploiting these facts we construct the structure element by selecting 6 speckles from an example image, and extracting a mask from these by selecting a threshold, such that the required shape is obtained. The structure element we use in the filter is the intersection of these 6 masks (see figure 4).

\section{FILTER}

The method proposed in this paper follows the new approach of first attempting to segment each individual speckle, after which each speckle is classified as a "noise speckle" or as a medically important resolvable detail:

Using the structure element of section 2 we first construct the morphological "opening" of the image. As is well known the opening of an image is obtained by first eroding the image with the structure element, after which one performs a dilation on this eroded image with the same structure element. The result of this operation 


\begin{tabular}{|l|l|l|l|l|l|l|l|l|l|l|l|}
\hline 0 & 0 & 0 & 0 & 0 & 0 & 0 & 0 & 0 & 0 & 0 & 0 \\
\hline 0 & 0 & 0 & 1 & 1 & 1 & 1 & 0 & 0 & 0 & 0 & 0 \\
\hline 0 & 1 & 1 & 1 & 1 & 1 & 1 & 1 & 1 & 1 & 1 & 0 \\
\hline 0 & 1 & 1 & 1 & 1 & 1 & 1 & 1 & 1 & 1 & 0 & 0 \\
\hline 0 & 0 & 0 & 0 & 0 & 0 & 0 & 0 & 0 & 0 & 0 & 0 \\
\hline
\end{tabular}

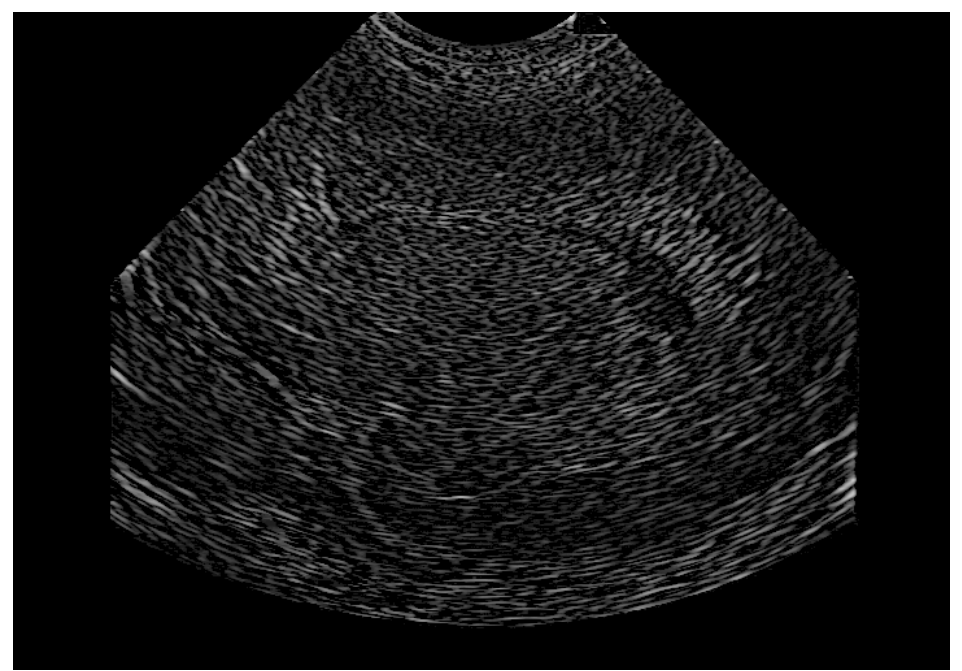

Figure 4: Left:Morphological structure element, Right: "top-hat transform" of the image in figure 6.

is an image in which the bright spots smaller than the structuring element are removed, while the dark spots are preserved. When we finally subtract this morphologically opened image from the original, we get the so-called "top-hat transform". This top-hat transform consists of the collection of foreground parts from the original image that fit the structure element (i.e., our "ideal speckle").

In the right-hand side of figure 4 we show an example of the result of applying the top-hat transform with the proposed structure element on the image of the left-hand side of figure 4.

After these pre-processing steps, we start segmenting the individual speckles. We start with the following datasets at our disposal: the top-hat transform of the original image, the compensated image (from the original image as it is produced by the ultrasound scanner), and a separate array, in which we can keep track of the speckles that are grown.

1) First we determine all local maxima in the original image above a prefixed lower threshold $\Lambda$, and put those in an array $M$.

2) Let $\Gamma$ be the highest of the grey-values of all elements in $M$. Choose one of those maxima $(i, j)$ with grey-value $\Gamma$. This pixel serves as a seed pixel for a region procedure, controlled by the grey-value of the pixels.

In short: a pixel $(m, n)$ belongs to the region of a seed pixel $(i, j)$, when the following criteria are satisfied:

- Pixel $(m, n)$ is "connected" to pixel $(i, j)$,

- $\alpha_{(m, n)}>\alpha_{(i, j)}-T$, where $T$ is a prefixed threshold,

- Pixel $(m, n)$ does not belong or is adjacent to a speckle which has already ben grown. In this way we segment one single speckle $\Sigma$.

3) Next we determine the centre of gravity $g_{\Sigma}$ of $\Sigma$, and consider the same position as $g_{\Sigma}$ in the compensated image. Take a square of $30 \times 30$ pixels around $g_{\Sigma}$ (in the compensated image), and calculate its mean grey-value $\mu_{\Sigma}$ and its contrast $\gamma_{\Sigma}$.

4)If $\mu_{\Sigma} \leq 67$ and $\gamma_{\Sigma} \leq 35$ then the pixel is located in healthy tissue, and hence is considered noise that should be removed. We calculate the mean $\nu_{\Sigma}$ of the grey-values of all the pixels adjacent to $\Sigma$, and give all pixel of $\Sigma$ the value $\nu_{\Sigma}$. In this way actually "cut off" the speckle.

5) If $\mu_{\Sigma}>67$ or $\gamma_{\Sigma}>35$, we conclude that the speckle is located in an affected part of the brain tissue, and hence the speckle $\Sigma$ need not be removed.

6) All local maxima (from $M$ ) that belong to $\Sigma$, or are adjacent to $\Sigma$, are removed from $M$. 


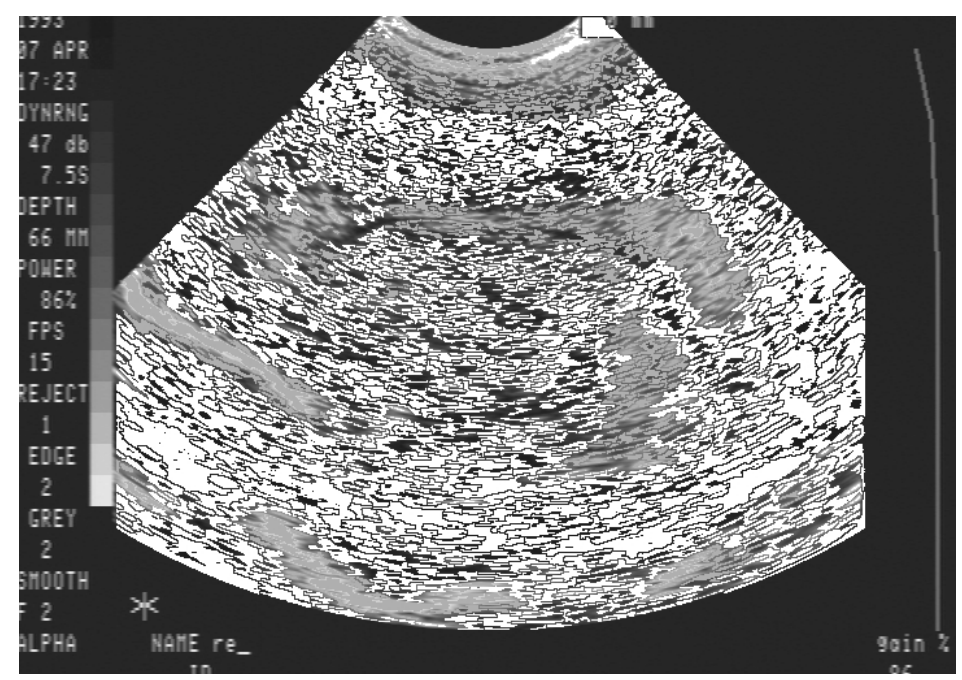

Figure 5: Classified speckles.

7) We repeat the whole process from step 2 until $M$ is empty.

Both $T$ and $\Lambda$ are adjustable parameters of the filter, and are dependent on the exact qualities of the ultrasound machine. By experiment, we obtained the best results with $\Lambda=63$ and $T=17$.

In figure 5 the speckles, as they are found by step 1), are indicated. The ones classified as "noise" (i.e., located in healthy tissue) are coloured white, the other ones are coloured grey.

\section{EXPERIMENTAL RESULTS}

We applied the proposed method on the image in figure 6 (left). The result is shown in figure 6 (right). In figure 7 we can find the manual delineation performed by an expert. We first notice that the speckle is suppressed strongly in the healthy tissue, while details are kept, and the affected tissue together with its internal structure is well-preserved. In figure 8 (left) we see the result of segmenting a flare on the original image with a so-called GVF-snake, ${ }^{17,18}$ starting from the position as depicted in figure 7. In figure 8 (right) the result is shown on the filtered image, starting from the same position. In the figures 9, 10 and 11 the results of the same experiment for a different image is exposed. Finally, in figure 12 we see the results of the same technique after the image has been filtered with respectively the Lee and the Frost filter.

\section{CONCLUSIONS}

In this paper we introduced a new speckle suppression technique for medical ultrasound images, which takes into account the morphological properties of speckle as well as quantitative, tissue characterizing, texture parameters. In our examples we achieved good speckle suppression in the healthy areas, while at the same we preserved important medical details. Visual inspection of the result shows clearly that the affected areas are better distinguishable,in particular the shape, area, and inner texture, which could serve as an aid in the diagnosis.

To validate our results, we segmented an affected area with a GVF-snake in the processed as well as the unprocessed image, thus using the filter as a pre-processing step for segmentation. Comparison of the results with the manual segmentation of an expert reveals a considerable improvement of the performance of the active contour when the image is processed with the proposed method. In this respect the proposed technique even outperforms the Lee filter and the Frost filter (two popular speckle suppression techniques). 

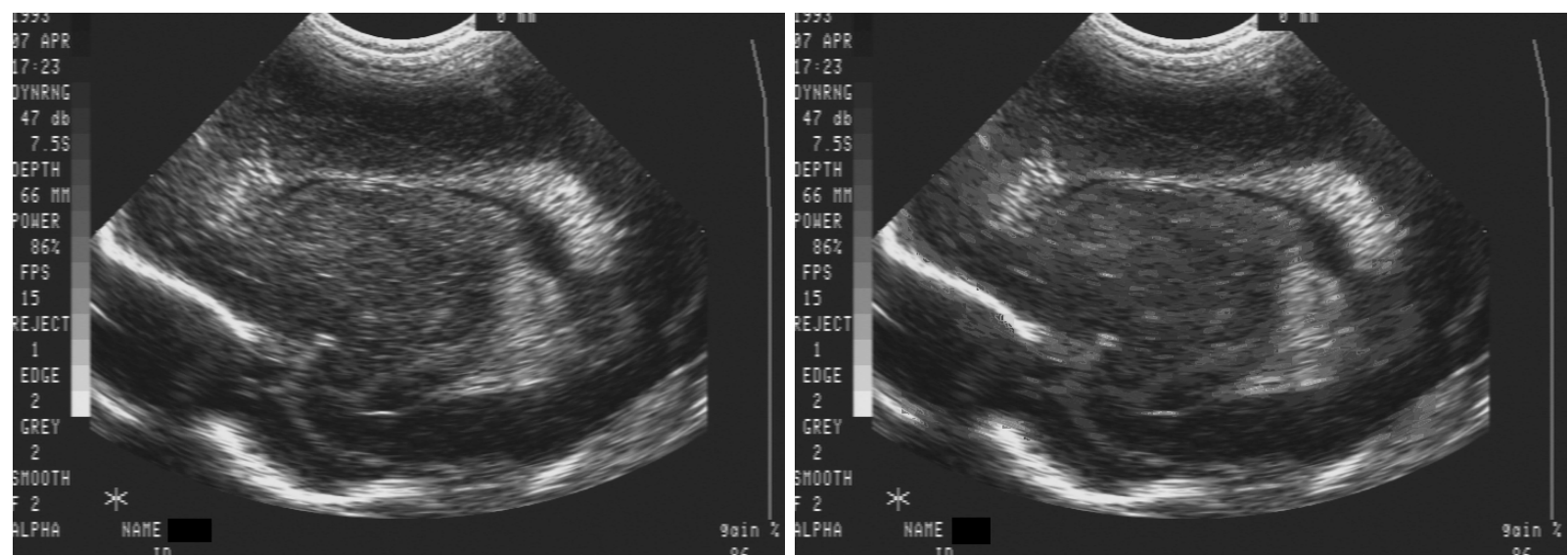

Figure 6: Left: Original image, Right: Filtered image.
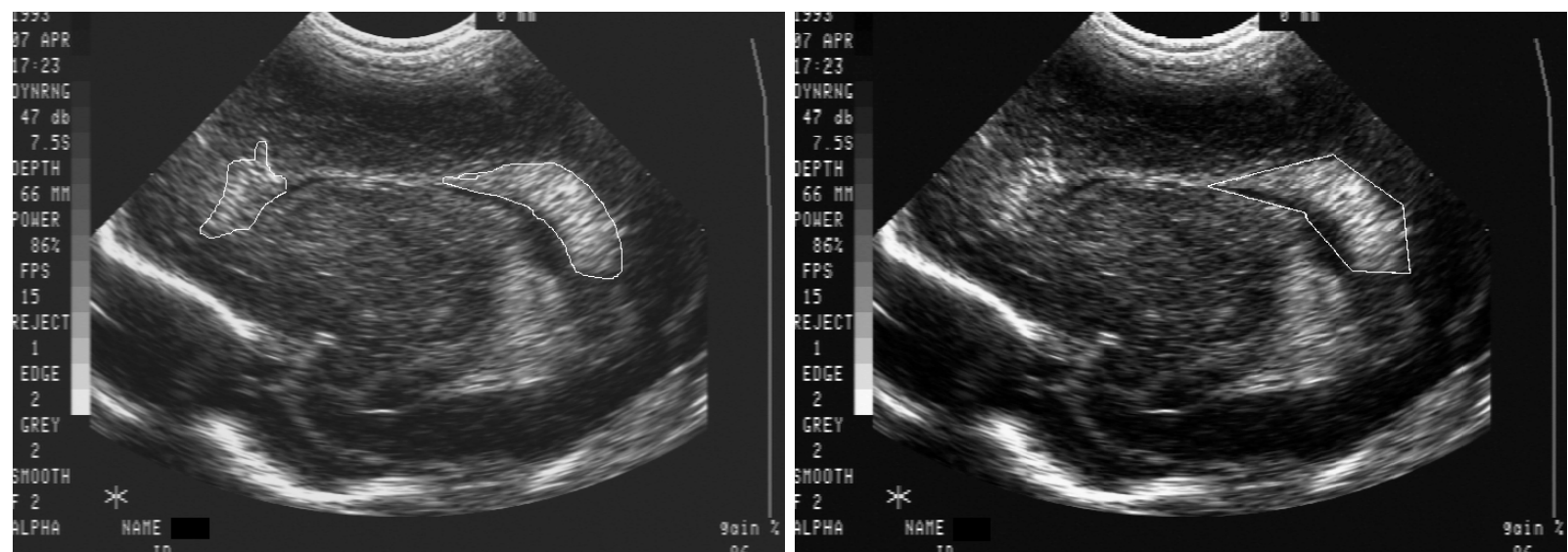

Figure 7: Left: Delineation by expert, Right: Initial position active contour.
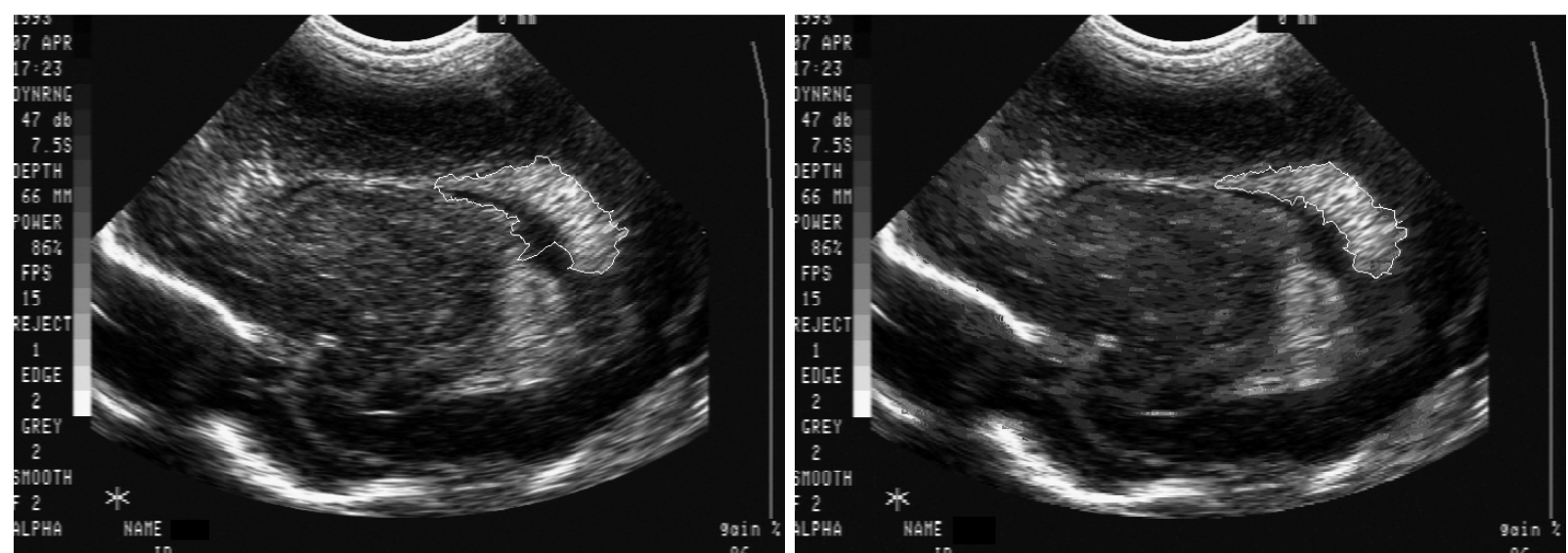

Figure 8: Left: Final state active contour on original image, Right: Final state on filtered image. 

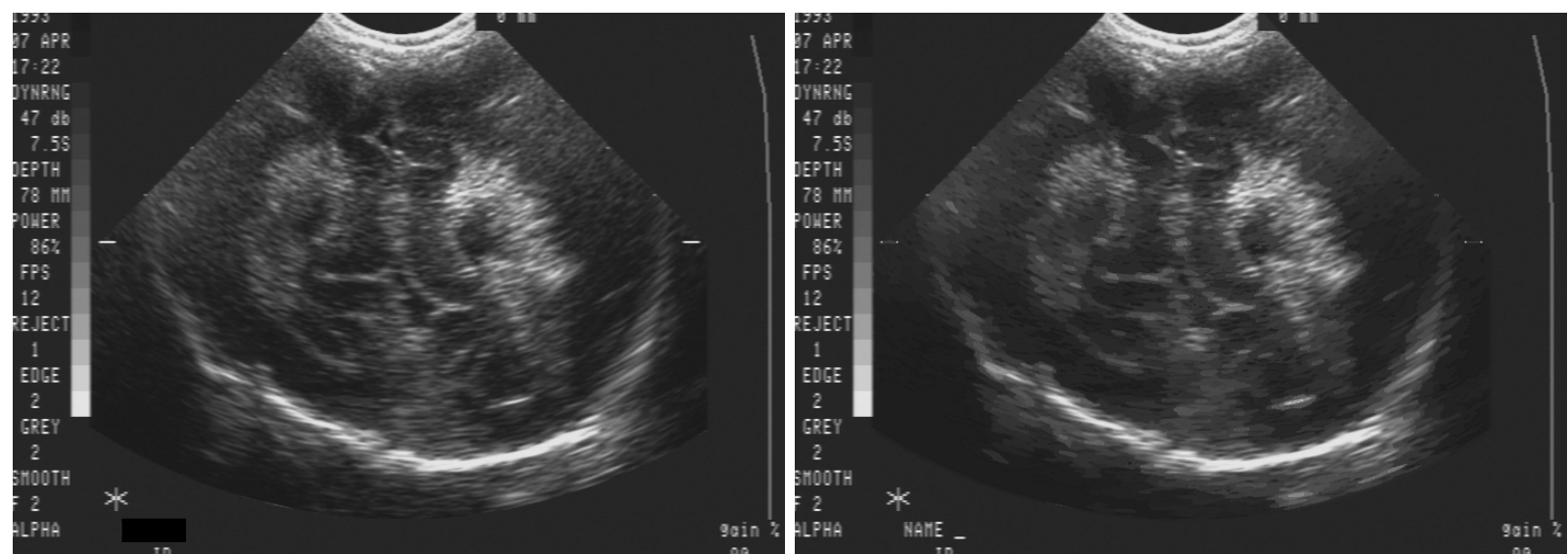

Figure 9: Left: Original image, Right: Filtered image.
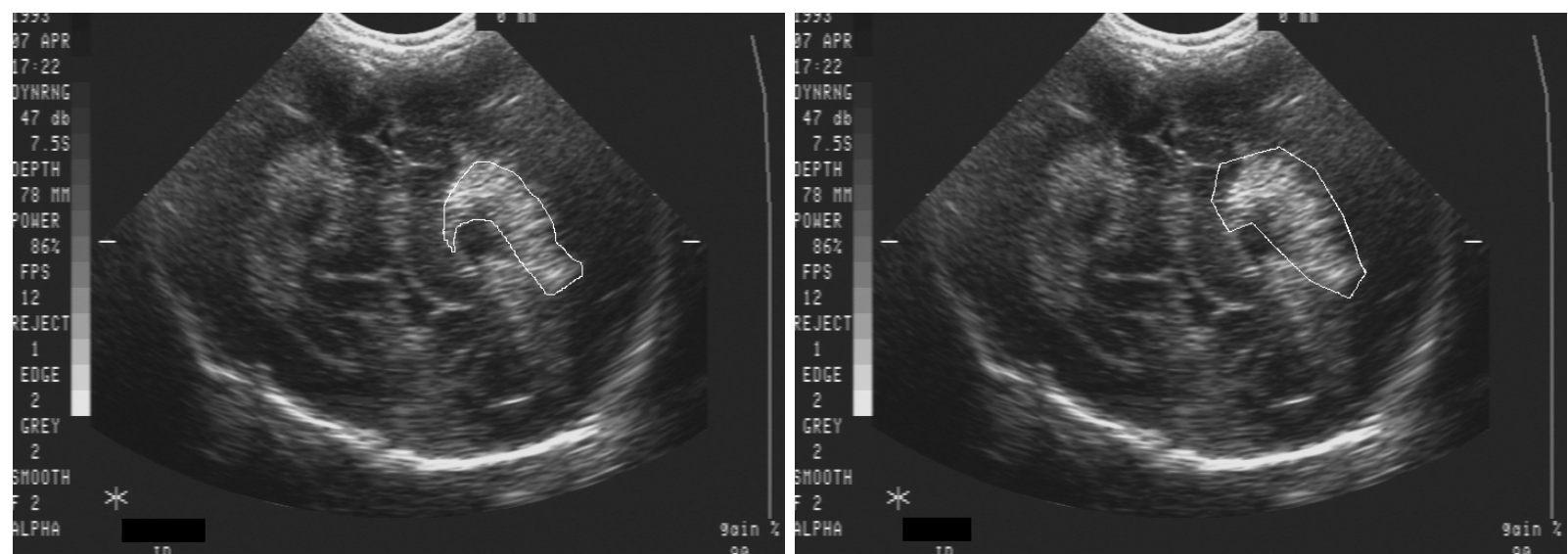

Figure 10: Left: Delineation by expert, Right: Initial position active contour.
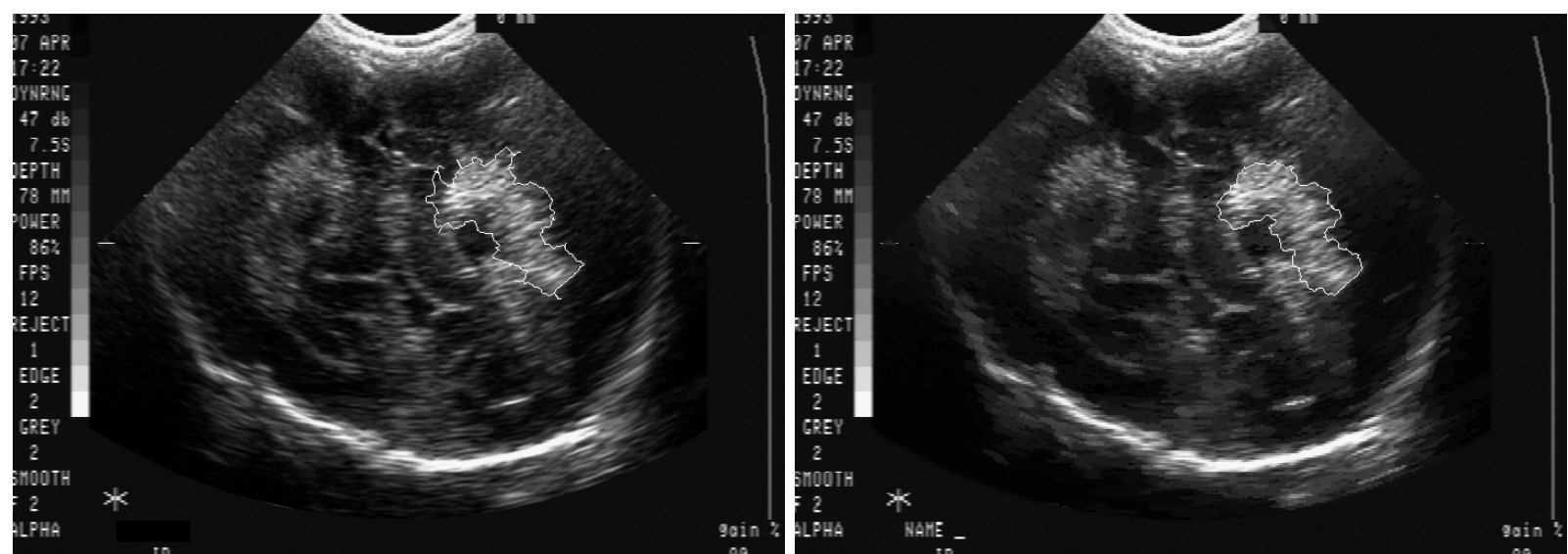

Figure 11: Left: Final state active contour on original image, Right: Final state on filtered image. 

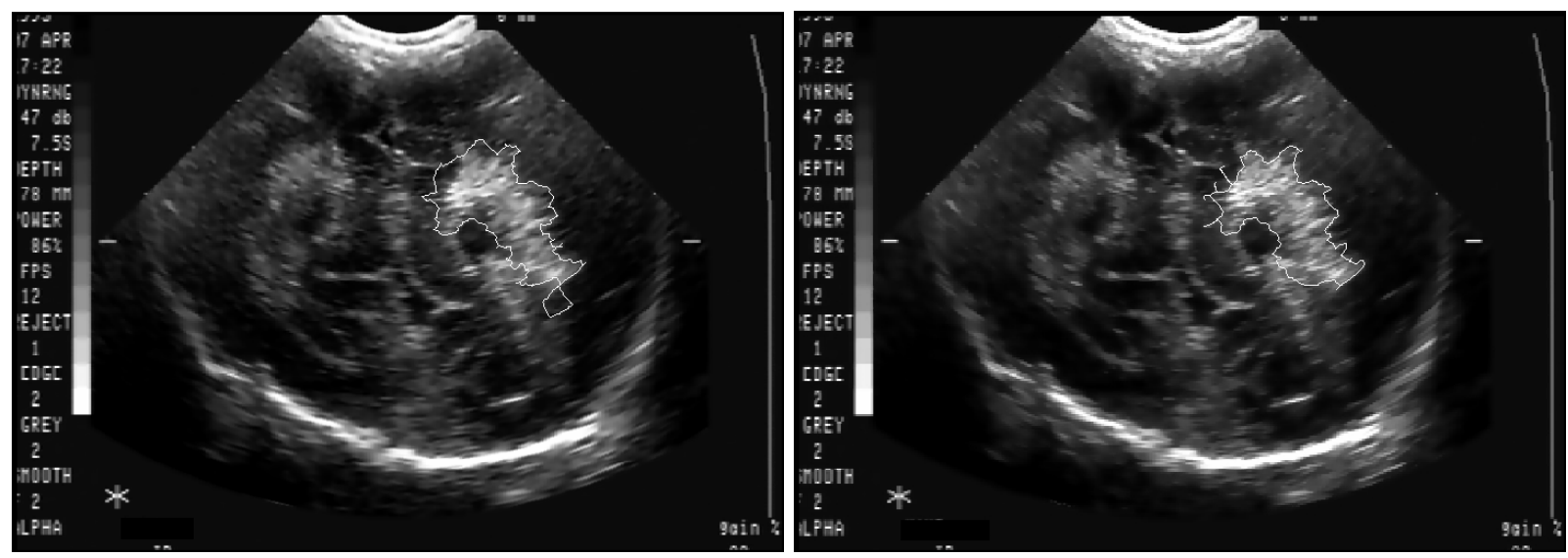

Figure 12: Left: Final state if filtered with Frost, Right: Final state if filtered with Lee.

\section{REFERENCES}

1. J. Lee, "Digital image enhancement and noise filtering by use of local statistics," IEEE Transactions on Pattern Analysis and Machine Intelligence 2(2), pp. 165-168, 1980.

2. V. Frost, J. Stiles, K. Shanmugan, and J. Holtzman, "A model for radar images and its application to adaptive digital filtering and multiplicative noise," IEEE Transactions on Pattern Analysis and Machine Intelligence 4, pp. 157-166, Mar. 1982.

3. M. Malfait and D. Roose, "Wavelet-based image de-noising using a markov random field a priori model," IEEE Transactions on Image Processing 6, pp. 549-565, Apr. 1997.

4. F. Sattar, L. Floreby, G. Salomonnson, and B. Lövström, "Image enhancement based on nonlinear multiscale method," IEEE Transactions on Image Processing 6, pp. 888-895, June 1997.

5. T. Loupas, W. N. McDicken, and P. L. Allan, "An adaptive weighted median filter for speckle suppression in medical ultrasonic images," IEEE Transactions on Circuits and Systems 36, pp. 129-135, Jan. 1989.

6. M. Karaman, M. Alper Kutay, and G. Bozdagi, "An adaptive speckle suppression filter for medical ultrasonic imaging," IEEE Transactions on Medical Imaging 14, pp. 283-292, June 1995.

7. P. Govaert and L. De Vries, An atlas of neonatal brain sonography, Clinics in Developmental Medicine, London: McKeith Press, 1996.

8. P. Govaert and L. De Vries, Geluiden uit de hersenen van de pasgeborene, St.-Luc, Nazareth, 1995.

9. B. Simaeys, W. Philips, I. Lemahieu, and P. Govaert, "Quantitative analysis of the neonatal brain by ultrasound," Computerized Medical Imaging and Graphics 24, pp. 11-18, 2000.

10. O. Basset, Z. Sun, J. Mestas, and G. Gimenez, "Texture analysis of ultrasonic images of the prostate by means of co-occurrence matrices," Ultrasonic Imaging 15, pp. 218-237, 1993.

11. Y. Kadah, A. Farag, J. Zurada, A. Badawi, and A. Youssef, "Classification algorithms for quantitative tissue characterization of diffuse liver disease from ultrasound images," IEEE Transactions on Medical Imaging 15(4), pp. 466-478, 1996.

12. Y.-N. Sun, H.-M. Horng, X.-Z. Lin, and J.-Y. Wang, "Ultrasonic image analysis for liver diagnosis," IEEE in Medicine and Biology, pp. 93-101, Nov./Dec. 1996.

13. G. Schmitz, H. Ermert, and T. Senge, "Tissue characterizations of the prostate using kohonen maps," in Proceedings of the IEEE Ultrasonics Symposium, pp. 1487-1490, 1994.

14. A. Huynen, R. Giessen, J. de la Rosette, R. Aamink, F. Debruyne, and H. Wijkstra, "Analysis of ultrasonographic prostate images for the detection of prostatic carcinoma: the automated urologic diagnostic expert system," Ultrasound in Medical Biology 20, pp. 1-10, 1994.

15. R. F. Wagner, S. W. Smith, and J. M. Sandrik, "Statistics of speckle in ultrasound b-scans," IEEE Trans. Son. Ultrason. 30, pp. 156-163, May 1983. 
16. J. M. Thijsen and B. J. Oosterveld, "Texture in tissue echograms; speckle or information?," J. Ultrasound Med. 19, pp. 215-229, 1990.

17. C. Zu and J. Prince, "Gradient vector flow: a new external force for snakes," in Conference on Computer Vision and Pattern Recognition (CVPR'97), pp. 66-71, 1997.

18. C. Zu and J. Prince, "Snakes, shapes, and gradient vector flow," IEEE Transactions on Image Processing 7, pp. 359-369, Mar. 1998. 